EOMmUn: Communication et organisation

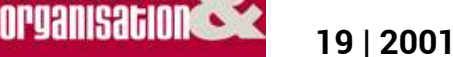

Actualité de la recherche en communication

\title{
Entretien avec Jacques Cosnier
}

Hugues Hotier

\section{OpenEdition}

Journals

Édition électronique

URL : http://journals.openedition.org/communicationorganisation/2537

DOI : 10.4000/communicationorganisation.2537

ISSN : 1775-3546

Éditeur

Presses universitaires de Bordeaux

Édition imprimée

Date de publication : 1 mai 2001

ISSN : 1168-5549

Référence électronique

«Entretien avec Jacques Cosnier », Communication et organisation [En ligne], 19 | 2001, mis en ligne le 27 mars 2012, consulté le 20 avril 2019. URL : http://journals.openedition.org/

communicationorganisation/2537 ; DOI : 10.4000/communicationorganisation.2537

Ce document a été généré automatiquement le 20 avril 2019.

(C) Presses universitaires de Bordeaux 


\title{
Entretien avec Jacques Cosnier
}

\author{
Hugues Hotier
}

\section{NOTE DE L'ÉDITEUR}

Propos recueillis par Hugues Hotier

1 Jacques Cosnier, docteur en médecine et docteur ès sciences, professeur émérite de psychologie des communications à l'Université Lumière (Lyon II) a pratiqué l'éthologie animale et humaine ainsi que la psychiatrie et la psychanalyse. Ancien Président de la Société Française d'Ethologie (SFECA), et ancien Président de l'association Applications des Recherches sur la Communication et les Interactions (ARCI), il est actuellement chercheur à l'unité de recherches associée au C.N.R.S. n 1347 (G.R.I.C.). Il est l'auteur ou co-auteur d'une dizaine de livres et d'une centaine d'articles dont: Les névroses expérimentales (Seuil), Clefs pour la psychologie (Seghers), Psychologie des sentiments et des émotions (Retz), Le retour de Psyché (Desclée de Brower).

Hugues Hotier: Jacques Cosnier, vous êtes un spécialiste reconnu du non-verbal, vous avez étudié le comportement de l'homme dans son milieu naturel et en ce sens le mot «éthologue » vous convient bien. Avant de parler de vos recherches et des méthodes que vous utilisez, peut-on en savoir un peu plus sur vous, sur vos origines, sur votre parcours?

3 Jacques Cosnier : Votre question est téméraire car j'ai commencé mes études de médecine en 1944...mon parcours professionnel s'étend donc sur plus de cinquante ans! Et en réalité ma vocation est encore antérieure: je désirais dans mon adolescence devenir éducateur et naturaliste, autrement dit professeur de sciences naturelles. La guerre me contraignit à entreprendre la médecine, qui se rapprochait le plus de cette ambition, cela peut expliquer qu'à la fin des années 50 je me trouvais Chef de travaux de Biologie en faculté de Médecine et spécialiste en Neuropsychiatrie. A partir de là et en résumant, je profitai de la création des enseignements de Psychologie à Lyon pour changer de Faculté : je passais à la Faculté des Sciences comme Maître-Assistant de Psychophysiologie. Or. le climat intellectuel de celte époque, je veux dire des années 50 et 60 était très effervescent: d'une part, et cela était net dans la partie expérimentalo-biologique. le béhaviorisme était Roi, d'autre part dans la partie socio-clinique, la Psychologie se 
séparait de la philosophie en empruntant à l'épanouissement du structuralisme des modèles qui agitaient les sciences humaines, en Anthropologie avec Levi-Stauss, en Sémiologie avec Barthes, en Psychanalyse avec Lacan, tandis qu'au même moment le mouvement antipsychiatrique se développait, et la découverte des drogues psychotropes révolutionnait les pratiques hospitalières... et aussi, last but not least, les Euvres des Ethologues Lorenz, Tinbergen étaient traduites en Français... Devant un tableau aussi riche il était difficile de choisir sa voie. Les neurosciences m'attiraient ainsi que l'expérimentation béhavioriste qui restait le modèle dominant de la Psychologie auto proclamée "scientifique", mais la pratique de la clinique aussi bien hospitalière que psychothérapique me montrait quotidiennement qu'il y avait un hiatus considérable entre les deux pôles. Tout mon savoir sur le système nerveux et sur la psychologie expérimentale ne me permettait pas de mieux comprendre et de mieux traiter les malades mentaux. Mon esprit naturaliste s'accordait beaucoup mieux avec les observations éthologiques. Ma thèse de sciences bien que comportant des aspects expérimentaux et même neurophysiologiques, s'intitulait "Étude éthologique du comportement grégaire du Rat de laboratoire »..., et ma thèse complémentaire sur « Les névroses Expérimentales " publiée en 1966 aux éditions du Seuil comparait les approches béhavioristes et les approches éthologiques elle annonçait clairement l'orientation qui allait devenir la mienne. Le Laboratoire que je créai alors s'appelait «Laboratoire de Psychologie Animale et Comparée » le "comparée » voulant dire que l'espèce humaine n'était pas exclue des recherches. Nommé professeur de Psychophysiologie, je dirigeais plusieurs thèses d'éthologie animale mais aussi de psychologie et de psychiatrie, l'éthologie humaine acquérait droit de cité, d'autant plus que Hubert Montagner de son côté développait une éthologie de l'enfant dans les crèches. Les réunions périodiques de nos laboratoires fondaient alors l'éthologie humaine en France, au sein de la société française d'éthologie (la SFECA) recueillant un gros succès de curiosité parmi les collègues aussi bien éthologues que béhavioristes. Pour en terminer avec ce curriculum j'ajouterai que les animaux non-humains étant remplacés progressivement clans mon laboratoire par l'Homo sapiens, le laboratoire prenait le nom de «Laboratoire d'Ethologie de Communications » et je changeais de faculté pour rejoindre la Psychologie proprement dite en tant que Professeur de Psychologie des Communications... Le groupe pluridisciplinaire de Recherches qui en est issu et auquel je suis encore rattaché s'appelle aujourd'hui «Groupe de Recherches sur les Interactions Communicatives ». C'est cette évolution de mon parcours: de la psychophysiologie aux interactions communicatives, qui me paraît significatif.

4 Hugues Hotier: On parle à votre propos, peut-être vous-même revendiquez-vous ce terme, d'éthologie de la communication. Pouvez-vous nous définir l'éthologie quand elle n'est pas l'étude du comportement animal. Et, pour être clair avec vous, je vous préviens que si vous parlez de comportement humain, je vous demanderai pourquoi il est nécessaire de parler d'éthologie quand le mot béhaviorisme existe depuis longtemps...

5 Jacques Cosnier : Oui je revendique ce terme. Mais votre question est justifiée : pourquoi l'éthologie? Alors que le béhaviorisme se définit déjà comme étude scientifique du comportement. Le béhaviorisme est un comportementalisme, comme d'ailleurs ce que signifie le mot anglais, et l'éthologie aussi ; mais comme je le soutenais dans mon livre de 1966 il y a une différence fondamentale entre les deux comportementalismes: le béhaviorisme est basé sur l'expérimentation, il se confond avec la S-R psychology, psychologie Stimulus-Réponse, c'est la psychologie des labyrinthes et des boîtes de 
Skinner, le comportement est étudié selon des plans expérimentaux classiques: hypothèse/soumission du sujet (animal ou humain) au protocole conçu pour tester l'hypothèse/résultats objectivables confirmant ou non l'hypothèse ; grâce à cela, ont été établies, entre autres les lois de l'apprentissage.

La perspective du comportementalisme éthologique est au moins au départ, très différente : le sujet, animal ou humain, est étudié dans son milieu naturel ou pour éviter des discussions inutiles, habituel. On ne se demande pas comment les sujets se comportent dans des dispositifs expérimentaux conçus par le chercheur pour confirmer ses idées préconçues, mais comment ils se comportent spontanément dans leur écologie familière. C'est l'observation qui va être fondamentale, observation souvent qualifiée de «naturaliste ». Les résultats obtenus sont très différents de ceux du béhaviorisme. Un chercheur béhavioriste peut avoir manipulé des milliers de Rats et tout ignorer des comportements reproducteurs et sociaux de ces animaux ainsi que la nature de leurs systèmes de communication, alors que ce sont des questions fondamentales pour l'éthologue. Bien sûr rien $\mathrm{n}^{*}$ empêche quand on connaît bien les mœurs d'une espèce d'expérimenter sur tel ou tel aspect du comportement. Mais souvent on utilisera alors la méthode des leurres, c'est à dire une méthode qui expose l'animal à des paramètres déduits de l'observation naturaliste.

Voilà ce qui répond à votre question, j'ajouterai cependant que le terme "éthologie " existe depuis plus longtemps que celui de " béhaviorisme ». il a été créé par les zoologues alors que le terme «béhaviorisme » est issu de la psychologie comparée.

Hugues Hotier: Pouvez-vous nous expliquer, voire nous décrire, la démarche de l'éthologie naturaliste? Si vous aviez un exemple, une application, à nous proposer, ce serait sans doute intéressant.

9 Jacques Cosnier : La démarche est facile à imaginer d'après ce qui précède. C'est une démarche de terrain. Par exemple on décide d'étudier le comportement des usagers du métro de Paris au niveau des bureaux de station (là où se trouvent les guichets de distribution des billets). Que s'y passe-t-il, quelles sont les paroles échangées entre l'agent et le client, etc...

10 Pour répondre à ces questions le chercheur va vivre dans le bureau de station, et dans un premier temps se familiarisera avec l'ambiance et le personnel, puis il installera son matériel d'enregistrement discret et enregistrera des échantillons d'interactions entre agents et usagers. On en tire plusieurs données : telles les différents types de demandes et corollairement les différentes fonctions assurées par les agents qui ne se limitent pas à la distribution des billets, telles aussi la pragmatique des échanges: ainsi le raccourcissement ou l'allongement de l'interaction verbale selon l'importance de la queue et de la compétence du voyageur etc. A partir de là on peut comparer avec ce qui se passe dans les bureaux d'information ou dans d'autres situations de guichet. Cette démarche d'observation naturaliste n'empêche pas de pratiquer en complément des entretiens avec les agents et les usagers et de tenir compte de leur vécu ou plus exactement de leurs façons de théoriser la situation. C'est pour cela que je parle parfois d'éthologie compréhensive car cette éthologie humaine rejoint évidemment l'ethnographie de la communication de Hymes et Gumperz, la microsociologie de Goffman et l'ethnométhodologie de Garfinkel. Goffman lui-même parlait d »éthologie de l'interaction. Sous cet angle on peut même soutenir que l'éthologie est plus une méthode ou une attitude de chercheur qu'une discipline autonome: c'est une discipline transversale. 
11 Je pourrais donner d'autres exemples, nous avons ainsi fait l'éthologie des interactions de soins en particulier celle d'un service hospitalier, l'éthologie d'un bureau de tabac, celle d'une grande rue de Lyon, d'une entreprise industrielle... enfin traditionnellement la psychologie du développement avec l'étude des interactions précoces utilise largement la méthode éthologique. Mais j'allais oublier un aspect important : si l'éthologie humaine est particulièrement fructueuse pour l'étude des interactions quotidiennes, elle l'est tout particulièrement pour l'étude de leurs aspects non-verbaux, ce qui fait parfois dire que les éthologues sont des spécialistes du non-verbal et qu'ils étudient l'homme comme s'il n'avait pas de langage, ce n'est qu'une boutade, car on peut faire évidemment aussi bien l'éthologie des interactions verbales que des non-verbales, et les deux sont d'ailleurs très intimement liées comme j'essaie de le démontrer depuis des années.

Hugues Hotier: Si je comprends bien, vous êtes bien loin de la sacro-sainte méthode hypothéticodéductive qui rassure tant les doctorants et les jurys de thèse. Cependant, on peut se demander si l'on n'a pas toujours une hypothèse quelque part dans la tête. Une hypothèse implicite. Sinon, comment justifier qu'on entreprenne une recherche quand il ne s'agit pas d'une commande?

13 Jacques Cosnier: C'est bien vrai que l'on est bien loin de la sacro-sainte méthode hypothético-déductive, mais chaque méthode a ses indications. Je pense que la méthode éthologique est particulièrement heuristique dans les approches où l'observation est essentielle par exemple dans la clinique, la psychologie du développement, la psychologie sociale, c'est-à-dire partout où les communications interindividuelles constituent un objet d'étude privilégié. Comment savoir ce qui se passe, ce qui se fait, ce qui se dit, sans aller sur le terrain?

Alors bien sûr se pose la question des hypothèses. Dans ce type d'approche on ne part pas d'hypothèses, on y aboutit. L'attitude de l'éthologue naturaliste rappelle l'attention flottante prescrite par Freud aux psychanalystes afin de réaliser un état de disponibilité réceptive exempte, autant que faire se peut, de présupposés. On a pu ainsi parler de la naïveté de l'éthologue c'est une naïveté méthodologique et volontaire dont certains candidats chercheurs sont d'ailleurs incapables.

Quant au problème de la justification d'une recherche non commanditée, c'est un problème qui n'est pas propre à ce type de recherche. Quelles sont les motivations profondes d'un chercheur? A quelle source s'alimente son épistémophilie? Il faut être certainement être un peu voyeuriste pour être passionné par l'observation de personnes ordinaires dans des activités quotidiennes banales.

Hugues Hotier: On sait que le chercheur qui pratique l'observation participante est en difficulté lorsqu'il s'agit d'interpréter puisqu'il n'est jamais assuré que ce qu'il a observé n'est pas le résultat de la perturbation qu'il a apportée au milieu dans lequel il s'est introduit. Comment l'éthologie naturaliste affronte-t-elle cette difficulté?

17 Jacques Cosnier : L'observation naturaliste n'est pas à proprement parler de l'observation participante, mais il est vrai comme l'ont soutenu les systémiciens qu'entrer dans un système n'est pas, qu'on le veuille ou non, sans effet sur le système et, on l'oublie souvent, n'est pas non plus sans effet sur celui qui s'y introduit.

18 Ceci dit, tous les terrains ne présentent pas la même difficulté, certains sont faciles, d'autres nécessitent des dispositifs techniques du genre caméra invisible. Si l'on veut énoncer un principe général, c'est celui de l'habituation nécessaire à la situation étudiée. Quand on fait de l'éthologie animale on va répétitivement sur le terrain et les animaux finissent par ne plus faire attention à cet animal familier et non dangereux qui vient 
hanter leur territoire, il en est de même en éthologie humaine, toute observation sérieuse devrait être précédée par une période d'imprégnation plus ou moins longue. C'est d'autre part durant cette période préliminaire que l'observateur fait connaissance avec les scénarios qu'il va étudier et dont il ne soupçonnait pas l'existence à priori.

Mais une difficulté propre à l'éthologie humaine c'est le fait d'appartenir à l'espèce que l'on étudie. Comment l'observateur peut-il gérer sa subjectivité et ses projections empathiques difficilement évitables? Hé bien, je crois qu'un premier pas essentiel est d'admettre que ce problème existe. Se faire une représentation de ce qui se passe en autrui, autrement dit interpréter sa conduite et inférer sur ses pensées implique qu'autrui a lui-même une activité de pensée et qu'il peut théoriser cette activité. Le mieux quand c'est possible est donc d'utiliser cela en lui demandant ce qu'il en pense. L'observation est ici participante du fait de la participation du sujet à la recherche. En éthologie animale on serait très heureux d'exploiter une telle possibilité et les efforts répétés pour faire parler les grands singes en sont la preuve. Alors pourquoi s'en priver avec le Primate bavard?

Enfin pour terminer sur la question de l'objectivité/subjectivité de l'observateur, on peut utiliser quand cela est nécessaire la méthode des juges, avec parfois la médiation d'informateurs : on montre à plusieurs experts les enregistrements avec un questionnaire ou avec des échelles d'évaluation et on mesure l'accord inter-juges.

En résumé je dirai que pour n'être pas expérimentale l'observation naturaliste n'en est pas moins rigoureuse. Elle n'est pas, non plus, plus facile, comme pourrait le faire croire une certaine littérature de vulgarisation, qui se pare de l'étiquette d'éthologie humaine, mais qui reprend simplement l'ambition triviale d'expliquer les comportements humains par les comportements animaux, lesquels étant eux-mêmes interprétés par projection des théories de l'auteur sur les comportements humains. Cette boucle stérile, qui recueille souvent un grand succès médiatique, est ce que l'on pourrait appeler de l'éthologie de salon, ou encore de l'éthologisme. Mais l'éthologie, il est vrai, n'est une appellation contrôlée.

Une bibliographie des problèmes abordés se trouve dans J. Cosnier «Le retour de Psyché », Desclée de Brouwer, 1998. 